\title{
ESTILOS DE APRENDIZAJE DE LOS ESTUDIANTES DE LA EDUCACIÓN SUPERIOR Y EL ACCESO A LAS HERRAMIENTAS DE ENTORNOS VIRTUALES
}

\author{
Higher education students' learning styles and access to virtual \\ environment tools
}

\author{
Paulo Alves \\ e-mail: palves@ipv.pt \\ Luisa Miranda \\ e-mail: Imiranda@ipb.pt \\ Carlos Morais \\ e-mail: cmmm@ipb.pt \\ Daniela Melaré \\ e-mail: dmelare@gmail.com \\ Instituto Politécnico de Bragança. Portugal
}

\section{RESUMEN}

El estudio se centra en la cuestión en torno de los estilos de aprendizaje de los estudiantes de la educación superior y el uso de herramientas digitales. Fue realizado con una muestra de 79 estudiantes de la educación superior, con el objetivo principal de determinar la influencia de los estilos de aprendizaje en el acceso a las herramientas del entorno virtual de una institución de educación superior, una plataforma soportada por el entorno colaborativo Sakai. A partir de los resultados obtenidos, se observa que la media más alta de las puntuaciones relacionadas con los estilos de los sujetos se encontró en el estilo reflexivo y que la herramienta que tuvo el mayor número de accesos fue la herramienta Recursos.

PALABRAS CLAVE: estilos de aprendizaje; CHAEA; educación superior; entorno virtual de aprendizaje; acceso a herramientas digitales.

\section{ABSTRACT}

This study focuses on the issue involving higher education students' learning styles and the use of digital tools. It was conducted with a sample of 79 undergraduates and its main aim was to determine the influence that learning styles may have on the access to the tools of the virtual environment of a higher education institution, a platform supported by the collaborative environment Sakai. In the light of the results obtained, it was found that the reflective style was the one which showed the highest mean of the scores obtained concerning the subjects' learning styles, and that the tool which showed the highest number of accesses was the Resources tool.

KEY WORDS: learning styles; CHAEA; higher education; virtual learning environment; access to digital tools.

Recibido/Received: 30/07/2017

Aprobado/Aproved: 23/12/2017

Cómo referenciar este artículo / How to reference this article:

Alves, P., Miranda, L., Morais, C., \& Melaré, D. (2018). Estilos de aprendizaje de los estudiantes de la educación superior y el acceso a las herramientas de entornos virtuales. Tendencias Pedagógicas, 31, 69-82. doi: http://dx.doi.org/10.15366/tp2018.31.003 


\section{INTRODUCCIÓN}

La diversidad y el aumento del número de estudiantes en la educación superior, y el hecho de que las realidades del mercado de trabajo están cambiando a un ritmo relativamente rápido plantean desafíos que caracterizan a la educación contemporánea y muestran cierto retraso en la formación académica para satisfacer las necesidades de los empleadores (Carol, 2015).

Truong (2016) sugiere que estudiantes diferentes tienen diferentes formas preferidas de aprendizaje. Algunos pueden entender rápidamente a través de imágenes, otros pueden preferir los textos, algunos pueden lidiar bien con las teorías, otros pueden aprender de las experiencias y ejemplos. Que tienen diferentes puntos de vista sobre los estilos de aprendizaje proporciona medios para la programación y el desarrollo de intervenciones adaptadas a las necesidades individuales de los estudiantes.

Las tecnologías de información y comunicación (TIC) y sus usos son particularmente importantes en las vidas de las personas cuando responden a sus necesidades, se ajustan a sus intereses y, en particular, a sus estilos de aprendizaje.

En este sentido, para determinar la influencia de los estilos de aprendizaje en el acceso a los recursos digitales, se desarrolló una investigación en una institución portuguesa de educación superior que ofrece a su comunidad académica uno entorno virtual, soportado por el entorno colaborativo Sakai, con varios recursos digitales de apoyo para las actividades desarrolladas por sus miembros, en particular las actividades de los profesores y estudiantes.

La importancia de esta investigación se justifica con la posibilidad de contribuir al desarrollo de estrategias que permitan mejorar el proceso de enseñanza y aprendizaje de los alumnos, teniendo en cuenta sus estilos de aprendizaje y los recursos digitales existentes, sobre todo los que están disponibles en los entornos virtuales de las instituciones a las que pertenecen. Los principales objetivos que guiaron la investigación son los siguientes:

- Caracterizar los estilos de aprendizaje de una muestra de estudiantes de educación superior;

- Identificar, en un semestre académico, la frecuencia con la que los estudiantes acceden a los recursos digitales disponibles en el entorno virtual;

- Determinar la relación entre los accesos a los recursos digitales disponibles en el entorno virtual y los estilos de aprendizaje de los estudiantes.

En la identificación de los estilos de aprendizaje de los sujetos de la muestra se utilizó el Cuestionario Honey-Alonso de Estilos de Aprendizaje (CHAEA), mientras que en la determinación de los accesos a los recursos digitales se utilizó el registro de los accesos efectuados en la plataforma durante un semestre académico. La frecuencia con la que los sujetos de la muestra acceden a los recursos digitales fue obtenida automáticamente por el entorno virtual. 
Estilos de aprendizaje de los estudiantes de la educación superior y el acceso a las herramientas de entornos virtuales

Además de la introducción, el artículo está organizado según los temas: fundamentos teóricos, metodología, resultados y discusión, y conclusiones, terminando con las referencias bibliográficas utilizadas en su elaboración.

\section{FUNDAMENTOS TEÓRICOS}

El aprendizaje es un proceso complejo y difícil. Varios parámetros, tales como la percepción y la construcción del conocimiento por los estudiantes, sus habilidades y sus características de desarrollo desempeñan un papel importante en este proceso. El proceso de aprendizaje se ve afectado por un número de diferentes factores que influyen en diferentes perspectivas de aprendizaje (Özyurt \& Özyurt, 2015).

Entre las competencias que los estudiantes de educación superior del siglo XXI deben dominar, se destacan las que están asociadas al conocimiento sobre la forma cómo aprenden. La capacidad de conocer su forma preferida de aprender y cómo aprenden en diferentes situaciones puede ser la respuesta a la necesidad continua de adaptarse a nuevas realidades (Gallego, 2013). Varios estudios indican que las personas tienen muchas diferencias individuales en la toma de decisiones, resolución de problemas y procesos de aprendizaje (Fatahi, Moradi, Kashani-Vahid, 2016).

El conocimiento de los estilos de aprendizaje de los estudiantes puede ayudar a mejorar la enseñanza y aprendizaje, puesto que los profesores pueden beneficiar de ese conocimiento para preparar materiales de apoyo al aprendizaje, incluyendo actividades que estén adecuadas a las formas de aprendizaje preferidas de los estudiantes, y de esta forma, haciendo el aprendizaje más fácil y atractivo para los estudiantes (Graf \& Kinshuk, 2010). Los estilos de aprendizaje se refieren a las diferencias individuales entre personas cuando inmersas en un proceso de aprendizaje (Moreno \& Defude, 2010).

Según Keefe, citado por Alonso, Gallego y Honey (1999), los estilos de aprendizaje son indicadores cognitivos, afectivos y fisiológicos relativamente estables de la forma cómo los estudiantes perciben, interactúan y responden en sus entornos de aprendizaje. Honey y Mumford (1992) consideran cuatro estilos de aprendizaje: activo, reflexivo, teórico y pragmático.

Las principales características de las personas asociadas a cada uno de los estilos de aprendizaje son las siguientes: estilo activo - animador, improvisador, descubridor, arriesgado y espontaneo; estilo reflexivo ponderado, concienzudo, receptivo, analítico y exhaustivo; estilo teórico metódico, lógico, objetivo, crítico y estructurado; estilo pragmático experimentador, práctico, directo, eficaz y realista (Alonso, Galego \& Honey, 1999).

Según Bender (2003), cuando se atienden los estilos de aprendizaje de los estudiantes y la enseñanza es orientada en función de los estilos de aprendizaje, los estudiantes pueden hacerse más responsables y alcanzar niveles más altos de aprendizaje. Gallego y Alonso (2010) afirman que la utilización del conocimiento de los estilos de aprendizaje de los estudiantes es esencial para mejorar el proceso de enseñanza y aprendizaje. 
Teniendo en cuenta la disponibilidad y el potencial de los recursos digitales en el apoyo al proceso de enseñanza y aprendizaje, representa un reto saber qué uso hacen los estudiantes de estos recursos y cuál es la relación entre ese uso y sus estilos de aprendizaje.

Con respecto a la utilización de los recursos digitales, en un estudio realizado por Miranda, Morais, Goulão y Melaré (2012), con una muestra de 147 sujetos de educación superior, se concluyó que la mayoría de los estudiantes de educación superior utiliza Internet todos los días. Señalan además que la gran mayoría de los estudiantes de educación superior utiliza Internet en su casa y en la universidad / trabajo para: búsqueda de información, fines educativos, comunicarse, trabajar, y para entretenimiento.

Una de las características personales que influye significativamente en la forma cómo los estudiantes perciben los entornos en línea y participan en ellos es su estilo de aprendizaje. La investigación en estilos de aprendizaje ofrece un importante conocimiento para aumentar los resultados académicos y el diseño de contenidos y estrategias de enseñanza (Küçük, Genç-Kumtepe, \& Tac, 2010).

Los estilos de aprendizaje se entienden como los rasgos personales que pueden influir en las experiencias de aprendizaje (Zacharis, 2011) y los resultados del aprendizaje en diferentes entornos. Graf y Liu (2010) identificó estilos de comportamiento del aprendizaje de los estudiantes en un curso online para desarrollar sistemas educativos adaptativos. La identificación de los recursos juega un papel importante en el sistema educativo y puede afectar el rendimiento y la eficiencia de la educación (Hung, Chang y Lin, 2016) estudiante.

En un estudio realizado por Graf, Liu y Kinshuk (2010) sobre el comportamiento de los estudiantes con respecto a la navegación en un sistema de gestión de aprendizaje en un curso en línea, los autores concluyeron que fueron identificadas varias diferencias en los patrones de navegación, lo que indica que los estudiantes con estilos de aprendizaje distintos utilizan distintas estrategias para aprender y navegar a través del curso.

Como resultado de un estudio sobre aspectos de utilización de herramientas digitales, Rodríguez, Lozano, Aradillas y Duque (2011) señalan que los estudiantes integrados en equipos con el mismo estilo predominante presentan percepciones favorables más altas con respecto al uso de la herramienta Google Docs y sus características que los estudiantes integrados en equipos de sujetos con estilos distintos.

Cela, Fuertes, Alonso y Sánchez (2010) señalan que los usuarios de herramientas web 2.0 prefieren utilizarlas en función de su estilo de aprendizaje y que la mayoría de las herramientas son utilizadas en actividades tanto personales como profesionales. Afirman además que las herramientas web 2.0 con una preferencia de utilización más alta son las de comunicación, blogs, wikis y video.

Como se ha señalado Truong (2016) los estilos de aprendizaje son fuentes útiles para el desarrollo de elearning sistemas adaptativos que personalizan los recursos de aprendizaje a las necesidades individuales de aprendizaje de los estudiantes. 


\section{METODOLOGÍA}

El estudio es de tipo cuantitativo, las variables asumen valores cuantitativos, y son exploradas relaciones entre las variables. Tal y como señala Creswell (2014), la investigación cuantitativa permite testar la relación entre variables y estas variables pueden ser medidas con recurso a instrumentos que fornecen datos numéricos que pueden ser analizados a través de procedimientos estadísticos. Para Kumar (2011), se puede considerar que un estudio es cuantitativo cuando se pretende cuantificar la variación de un fenómeno, una situación, problema o cuestión, la información es obtenida a través de variables esencialmente cuantitativas, y cuando el análisis de la información está orientado para determinar la magnitud de su variación.

La recolección de datos obedeció a dos procesos distintos, según los datos se relacionan con los estilos de aprendizaje o con el acceso a los recursos digitales. Los dos procesos fueron soportados por el entorno virtual y resultaron en una recolección automática de datos en función de la acción de los sujetos de la muestra.

Los datos relacionados con la caracterización de los estilos de aprendizaje fueron obtenidos en el entorno virtual, a través de la compleción del cuestionario CHAEA, lo cual constituye una de las herramientas del entorno virtual. Los datos relacionados con el número de accesos a los recursos digitales fueron registrados automáticamente cada vez que los sujetos de la muestra accedían a cada uno de los recursos.

La muestra invitada se compone de todos los estudiantes de dos escuelas de educación superior pública, siendo una de las escuelas de Educación y la otra de Tecnología y Gestión, con aproximadamente 3800 estudiantes. Entre estos, 79 sujetos constituyeron la muestra que forneció los datos para este estudio completando el cuestionario CHAEA y accediendo a recursos digitales. De los 79 sujetos, $77,2 \%$ son del género femenino y $22,8 \%$ del género masculino. Con respecto a la escuela a la que pertenecen, $50,6 \%$ son de la Escuela Superior de Educación y 49,9\% de la Escuela Superior de Tecnología y Gestión.

Las variables estudiadas están asociadas a estilos de aprendizaje y a recursos digitales. Las que están asociadas a los estilos son: Activo, Reflexivo, Teórico y Pragmático, y cada una de ellas asume valores enteros que varían de cero a 20 puntos.

A las variables asociadas a los recursos digitales se les dieron los mismos nombres con los que están identificadas en el entorno virtual, es decir: Fórums, Mensajes, Recursos, Testes En Línea y Trabajos. Cada una de las variables asume valores que varían de cero a el número máximo de accesos realizado por el estudiante que accedió más veces al recurso.

En lo que concierne al tratamiento de datos, se destaca el análisis descriptivo, organizando los datos en cuadros y gráficos, así como el análisis inferencial a través de la utilización del programa estadístico SPSS para determinar los coeficientes de correlación de Spearman entre las variables bajo estudio. 


\section{RESULTADOS Y DISCUSIÓN}

En la presentación de los resultados se destacan los aspectos que pueden contribuir a alcanzar los objetivos propuestos para esta investigación. Así pues, se presenta la caracterización de los estilos de los sujetos de la muestra y la relación entre los estilos de aprendizaje y el acceso a recursos digitales.

\subsection{Caracterización de los Estilos de Aprendizaje de los Sujetos de la Muestra}

La identificación de los estilos de aprendizaje de los sujetos que participaron en la investigación fue realizada a partir de la compleción del cuestionario CHAEA disponible en el entorno virtual utilizado en la institución donde se realizó el estudio. El cuestionario CHAEA (Cuestionario HoneyAlonso de Estilos de Aprendizaje) se compone de 80 ítems breves y dicotómicos, cada 20 correspondiendo a un estilo de aprendizaje: Activo, Reflexivo, Teórico y Pragmático. Con respecto a la puntuación, la respuesta a cada ítem admite la puntuación de uno o cero puntos, según se está más o menos de acuerdo, respectivamente, con el perfil definido por la teoría de los estilos para cada estilo. Asimismo, hay en el cuestionario preguntas relacionadas con la caracterización de quien lo completa e instrucciones sobre su compleción, a saber un pedido para que se completen todos los ítems del cuestionario, y que la respuesta se traduce por el signo "+" según se está más de acuerdo con la afirmación del ítem o por el signo "-" cuando se está más en desacuerdo que de acuerdo con la afirmación constante en el ítem bajo análisis. Las 20 cuestiones relacionadas con cada estilo están distribuidas de forma aleatoria por el cuestionario para que el respondiente no pueda identificar los ítems asociados a cada estilo (Miranda \& Morais, 2008).

En la caracterización del nivel de preferencia de los sujetos de la muestra en cada estilo de aprendizaje, se utilizaron los criterios definidos por Alonso, Gallego y Honey (1999), presentados en el Cuadro 1.

Cuadro 1: Preferencias en los estilos de aprendizaje

(Adaptado de Alonso, Gallego \& Honey, 1999, p.114)

\begin{tabular}{|l|c|c|c|c|c|}
\hline \multirow{2}{*}{$\begin{array}{c}\text { Estilos de } \\
\text { Aprendizaje }\end{array}$} & \multicolumn{5}{|c|}{ Niveles de Preferencia } \\
\cline { 2 - 6 } & Muy Baja & Baja & Moderada & Alta & Muy Alta \\
\hline Activo & $0-6$ & $7-8$ & $9-12$ & $13-14$ & $15-20$ \\
\hline Reflexivo & $0-10$ & $11-13$ & $14-17$ & $18-19$ & 20 \\
\hline Teórico & $0-6$ & $7-9$ & $10-13$ & $14-15$ & $16-20$ \\
\hline Pragmático & $0-8$ & $9-10$ & $11-13$ & $14-15$ & $16-20$ \\
\hline
\end{tabular}

Se presupone que uno de los objetivos de la identificación de los estilos de aprendizaje de los estudiantes consiste en promover el autoconocimiento de las características cognitivas, afectivas y fisiológicas que permiten a cada estudiante comprender su forma de aprender, interactuar y responder en los 
entornos de aprendizaje. Así pues, cada estudiante podrá beneficiar de ese conocimiento para mejorar sus procesos de aprendizaje, conociendo los indicadores en los que está mejor y aquellos en los que necesita mejorar. De esta forma, cuanto más cerca de 20 sea la puntuación del estudiante en cada uno de los estilos de aprendizaje, más preparado estará para aprender. Para que cada estudiante pueda identificar su nivel de preferencia en cada estilo de aprendizaje, cada uno recibe del entorno virtual, después de haber completado el cuestionario CHAEA en ese mismo entorno, un feedback como el que se presenta en la Figura 1.

\section{Resultado·del-cuestionario·Honey-Alonso-CHAEAT}
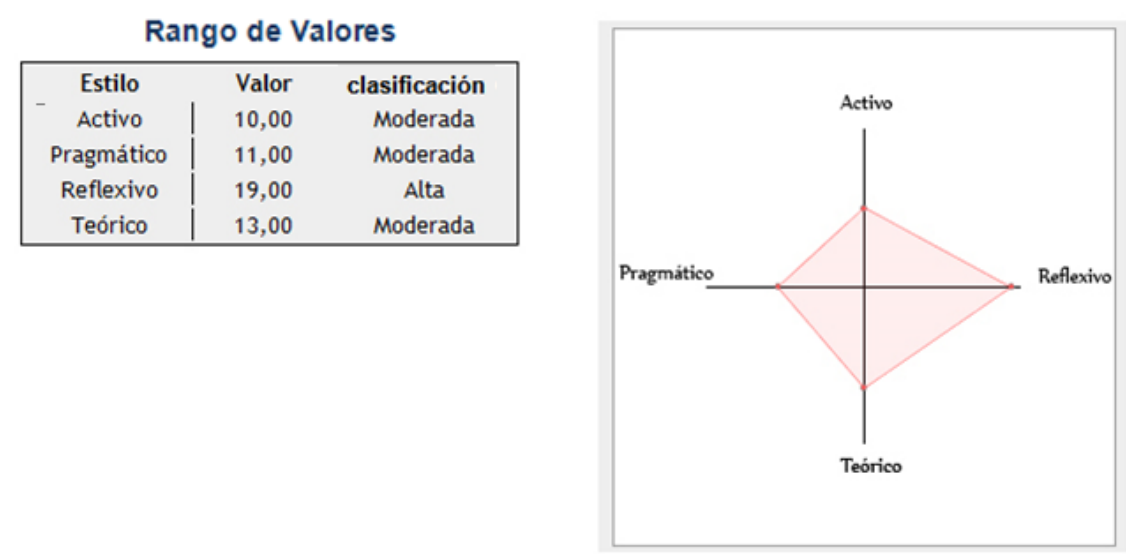

Figura 1 - Feedback enseñado al estudiante después de completar el cuestionario CHAEA

El estudiante que tenga un perfil como el que está presentado en la Figura 1 está bastante bien en los aspectos asociados al estilo reflexivo puesto que obtuvo 19 puntos en 20. Por otro lado, necesita mejorar bastante en los aspectos asociados a otros estilos. Así pues, resulta importante evidenciar los aspectos relevantes de cada estilo de aprendizaje que ayudan a que el estudiante mejore su perfil de aprendizaje, para que quede claro para quien completa el cuestionario CHAEA cuáles son las características que tiene ya consolidadas y cuáles necesita mejorar.

Además de las ventajas de las que cada sujeto pueda beneficiar por conocer su propio perfil de aprendizaje, también es importante para los profesores conocer el perfil predominante de los estudiantes que integran los cursos que enseñan, puesto que, de esa forma, podrán diseñar e implementar estrategias de enseñanza y aprendizaje que satisfagan más fácilmente los intereses y formas de aprender de sus estudiantes. Si se toma la muestra como un todo, se puede considerar como si se tratara de un curso superior. Así pues, teniendo en cuenta la escala utilizada para cada estilo, de cero a 20 puntos, y para que se puedan relacionar las características de la muestra en función de los estilos de aprendizaje y la utilización de herramientas digitales, se presenta en el Cuadro 2 la distribución de los sujetos de la muestra en lo que concierne a los estilos de aprendizaje y respectivos niveles de preferencia. Puesto que cada sujeto puede admitir preferencias iguales en más de un estilo, en general 
el número de sujetos en cada nivel de preferencia es superior al tamaño de la muestra, o sea, superior a 79 .

Cuadro 2: Niveles de preferencia en los estilos de aprendizaje

\begin{tabular}{|l|c|c|c|c|c|c|c|c|}
\hline \multirow{2}{*}{ Niveles de preferencia } & \multicolumn{2}{|c|}{ Activo } & \multicolumn{2}{|c|}{ Reflexivo } & \multicolumn{2}{c|}{ Teórico } & \multicolumn{2}{c|}{ Pragmático } \\
\cline { 2 - 9 } & $\mathrm{n}$ & $\%$ & $\mathrm{n}$ & $\%$ & $\mathrm{n}$ & $\%$ & $\mathrm{n}$ & $\%$ \\
\hline Muy Alta & 21 & 26,6 & 7 & 8,9 & 19 & 24,1 & 21 & 26,6 \\
\hline Alta & 19 & 24,1 & 15 & 19,0 & 18 & 22,8 & 26 & 32,9 \\
\hline Moderada & 32 & 40,5 & 35 & 44,3 & 34 & 43,0 & 22 & $27,8 \%$ \\
\hline Baja & 5 & 6,3 & 15 & 19,0 & 6 & 7,6 & 8 & $10,1 \%$ \\
\hline Muy Baja & 2 & 2,5 & 7 & 8,9 & 2 & 2,5 & 2 & $2,5 \%$ \\
\hline
\end{tabular}

Tras analizar el Cuadro 2, se puede observar que el nivel de preferencia moderada es el que tiene un porcentaje más alto de sujetos en los estilos Activo, Reflexivo y Teórico, mientras que en el estilo Pragmático, la preferencia con el porcentaje más alto es la preferencia alta. Con niveles de preferencia alta o muy alta existen $51,7 \%$ en el estilo Activo, $27,9 \%$ en el estilo Reflexivo, $46,9 \%$ en el estilo Teórico y $59,5 \%$ en el estilo Pragmático. Con niveles de preferencia baja o muy baja existen $8,8 \%$ en el estilo Activo, $27,9 \%$ en el estilo Reflexivo, $10,1 \%$ en el estilo Teórico y $12,6 \%$ en el estilo Pragmático. Estos datos permiten inferir que los sujetos de la muestra necesitan mejorar el perfil asociado al estilo Reflexivo, puesto que es en éste que se observa el porcentaje más bajo en lo concierne a los niveles de preferencia alta o muy alta.

En el Cuadro 3 se presenta una síntesis descriptiva de los datos sobre las puntuaciones obtenidas por los sujetos de la muestra en cada uno de los estilos de aprendizaje.

Cuadro 3: Estadísticas de las puntuaciones obtenidas en los estilos de aprendizaje $(n=79)$

\begin{tabular}{|l|c|c|c|c|c|}
\hline Estilos de aprendizaje & Mínimo & Máximo & Media & Mediana & Moda \\
\hline Activo & 5 & 18 & 12,4 & 13 & 12 \\
\hline Reflexivo & 7 & 20 & 15,2 & 16 & 16 \\
\hline Teórico & 6 & 20 & 13,4 & 13 & 13 \\
\hline Pragmático & 8 & 20 & 13,8 & 14 & 14 \\
\hline
\end{tabular}

Los datos presentados en el Cuadro 3 revelan que el máximo de las puntuaciones no fue logrado solamente en el estilo Activo, además de que es en este mismo estilo que se observa el mínimo de las puntuaciones y la media más baja de puntuaciones. Por otro lado, es en el estilo Reflexivo que se observa a media $(15,2)$ y la moda (16) de puntuaciones más altas. 


\subsection{Relación Entre los Estilos de Aprendizaje y el Acceso a Recursos Digitales}

En el sentido de mejorar las estrategias de enseñanza y aprendizaje teniendo en cuenta los estilos de aprendizaje de los estudiantes, se dio particular destaque a la relación entre los estilos predominantes en los estudiantes y la frecuencia de acceso y utilización de las herramientas del entorno virtual, a saber Fórums, Mensajes, Recursos, Testes en Línea y Trabajos.

En el Cuadro 4 se presenta una síntesis descriptiva de los datos sobre el acceso de los sujetos de la muestra a los recursos digitales disponibles en el entorno virtual.

Cuadro 4: Accesos a herramientas del entorno virtual

\begin{tabular}{|l|c|c|c|c|c|}
\hline \multirow{2}{*}{ Herramientas } & \multicolumn{5}{|c|}{ Accesos (3732) } \\
\cline { 2 - 6 } & N.o & $\%$ & N.․ Máximo & ․o. Mínimo & Media \\
\hline Fórums & 154 & 4 & 40 & 0 & 1,9 \\
\hline Mensajes & 232 & 6 & 12 & 0 & 2,9 \\
\hline Recursos & 3297 & 88 & 251 & 0 & 41,7 \\
\hline Testes En Línea & 27 & 1 & 5 & 0 & 0,3 \\
\hline Trabajos & 22 & 1 & 6 & 0 & 0,3 \\
\hline
\end{tabular}

Los resultados presentados en el Cuadro 4 se evidencian en la Figura 2.

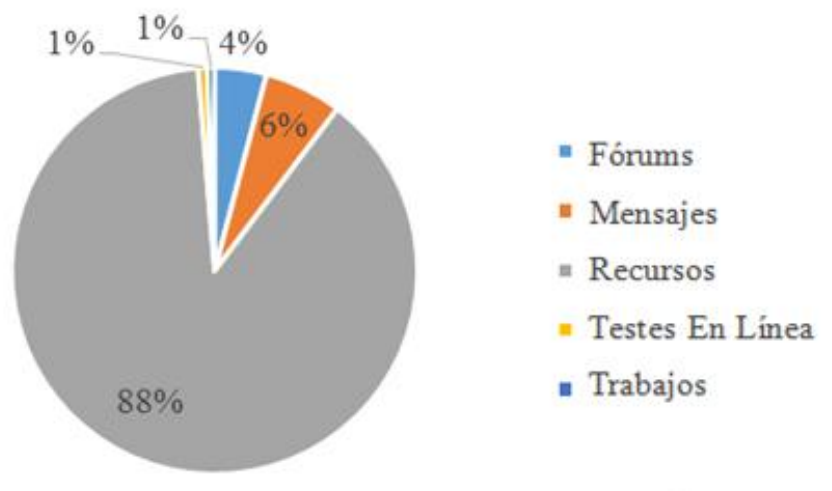

Figura 2: Acceso a las herramientas del entorno Sakai (3732 accesos)

El análisis del Cuadro 4 permite observar que la herramienta con un número más alto de accesos es la herramienta Recursos, mientras que es prácticamente irrelevante el uso que los estudiantes hacen de las otras herramientas. Este hecho obliga a una reflexión profunda sobre el uso que los estudiantes hacen de los recursos digitales. No es suficiente que estén disponibles, es imprescindible incentivar a los estudiantes para su utilización y particularmente llevarlos a admitir que la utilización de recursos digitales puede mejorar su rendimiento escolar. 
Tras haber identificado la frecuencia de los accesos a las herramientas digitales, buscamos comprender si ese acceso es influenciado por el perfil de los estudiantes en lo que concierne a los estilos de aprendizaje. Para eso, se procedió a la identificación de los coeficientes de correlación entre las variables.

Según Morais (2000), considerando el coeficiente de correlación (ठ), la correlación entre dos variables puede ser clasificada de la siguiente manera: muy alta, si $\delta \in([-1 ;-0,9] \cup[0,9 ; 1])$; alta, si $\delta \in(]-0,9 ;-0,7] \cup[0,7 ; 0,9[)$; moderada, si $\delta \in(]-0,7 ;-0,4] \cup[0,4 ; 0,7[)$; baja, si $\delta \in(]-0,4 ;-0,2] \cup[0,2 ; 0,4[)$; e muy baja, si $\delta \in]-0,2 ; 0,2[$.

Las variables relacionadas con los etilos de aprendizaje, puntuaciones obtenidas por cada sujeto en los estilos Activo, Reflexivo, Teórico y Pragmático, así como las variables relacionadas con la frecuencia de acceso a los recursos digitales, asociadas al número de veces que accedieron a Fórums, Mensajes, Recursos, Testes En Línea y Trabajos, son variables cuantitativas. Tal y como afirma Pereira (2004), el grado de asociación entre las variables puede ser determinado utilizando el coeficiente de correlación de Pearson cuando la distribución de las variables es normal, o a través del coeficiente de correlación de Spearman cuando la distribución de las variables no es normal.

Así pues, de manera a determinar si la distribución de las variables es normal, se utilizó el test de Kolmogorov - Smirnov (K-S) (Maroco, 2010). La aplicación del test K-S a través de SPSS permitió observar que solamente las variables asociadas a los estilos de aprendizaje Activo, Reflexivo, Teórico y Pragmático admiten una distribución normal, mientras que las variables Fórums, Mensajes, Recursos, Testes en Línea y Trabajos no admiten una distribución normal. Consecuentemente, las distribuciones de las variables bajo estudio no cumplen las condiciones de aplicabilidad de la correlación de Pearson, lo que implicó optar por la determinación de los coeficientes de correlación de Spearman (Pereira, 2004).

Los resultados de la determinación de los coeficientes de correlación de Spearman se encuentran presentados en el Cuadro 5.

Cuadro 5: Coeficientes de correlación de Spearman entre las variables asociadas a los estilos de aprendizaje y las asociadas al acceso a recursos digitales $\left({ }^{* *}\right)$

\begin{tabular}{|l|l|l|l|c|c|c|}
\hline \multicolumn{2}{|l|}{ Estilos } & Fórums & Mensajes & Recursos & $\begin{array}{c}\text { Testes En } \\
\text { Línea }\end{array}$ & Trabajos \\
\hline \multirow{2}{*}{ Estilo Activo } & Correlación & 0,109 & 0,039 & 0,054 & $-0,073$ & 0,055 \\
\cline { 2 - 7 } & Nivel Sig. & 0,341 & 0,736 & 0,634 & 0,524 & 0,630 \\
\hline \multirow{2}{*}{ Estilo Reflexivo } & Correlación & 0,109 & $-0,151$ & $-0,076$ & $-0,142$ & 0,172 \\
\cline { 2 - 7 } & Nivel Sig. & 0,341 & 0,185 & 0,505 & 0,212 & 0,130 \\
\hline \multirow{2}{*}{$\begin{array}{l}\text { Estilo Teórico } \\
\text { Estilo } \\
\text { Pragmático }\end{array}$} & Correlación & 0,190 & $-0,229^{*}$ & $-0,028$ & $-0,111$ & 0,164 \\
\cline { 2 - 7 } & Nivel Sig. & 0,093 & 0,043 & 0,808 & 0,332 & 0,148 \\
\cline { 2 - 7 } & Nivel Sig. & 0,093 & 0,178 & 0,154 & 0,125 & 0,066 \\
\hline
\end{tabular}

*. La correlación es significativa en el nivel 0,05 (bilateral) 
${ }^{* *} \mathrm{n}=79$ en todas las situaciones

Tras observar los datos presentados en el Cuadro 5 , y teniendo en cuenta la clasificación definida por Morais (2000), así como los valores encontrados para los coeficientes de correlación a través de la aplicación del test de Spearman, se evidencia que la correlación sólo es baja entre las variables estilo Teórico y Mensajes, con un nivel de significancia inferior 0,05. En las demás situaciones, no existe correlación significativa con un nivel de significancia inferior a 0,05 entre ninguna de las parejas de variables analizadas, por lo que no se clasificará la respectiva correlación en función del coeficiente de correlación encontrado.

Así pues, a partir de los datos obtenidos, se puede afirmar que los estilos de aprendizaje de los sujetos de la muestra no influyen significativamente en el número de accesos de cada estudiante a los recursos digitales.

\section{CONCLUSIONES}

El estudio fue realizado con una muestra de 79 estudiantes de una institución de educación superior portuguesa. Con este estudio, se trató de determinar las características de los sujetos de la muestra en lo que concierne a los estilos de aprendizaje utilizando la clasificación de los estilos propuesta por Honey-Alonso, es decir activo, reflexivo, teórico y pragmático y admitiendo para cada estilo los niveles de preferencia muy baja, baja, moderada, alta y muy alta.

En una escala de cero a veinte puntos para cada estilo, las medias de puntuaciones variaron entre 12,4 en el estilo Activo y 15,2 en el estilo Reflexivo, siendo de 13,4 en el estilo Teórico y de 13,8 en el estilo Pragmático. Los datos no permitieron la construcción de grupos independientes con características muy distintas en función de los estilos de los sujetos de la muestra.

Con respecto a las herramientas Fórums, Mensajes, Recursos, Testes en Línea y Trabajos, utilizadas durante un semestre académico en entorno virtual, una plataforma soportada por el entorno colaborativo Sakai, se observó que el número de accesos de la muestra varió entre 22 en la herramienta Trabajos y 3297 en la herramienta Recursos, siendo que el número de accesos a la herramienta Recursos corresponde a un $88 \%$ de la totalidad de los accesos. Así pues, se puede concluir que la única herramienta utilizada con una frecuencia bastante alta es la herramienta Recursos.

Esta herramienta es el tipo que esta relacionado a todos los estilos de aprendizaje y sus facilidades, de acuerdo con las investigaciones de Alves, Barros e Okada (2009) es importante tener en cuenta que las interfaces se repiten en los estilos de aprendizaje y cada uno se complementan con otros que difieren en su conjunto. El más importante es el camino pedagógico disponible de acuerdo a las preferencias individuales de los estilos.

En lo concierne a la relación entre el número de accesos a recursos digitales y los estilos de aprendizaje, se utilizó el programa SPSS y los coeficientes de correlación de Spearman. No fueron encontradas evidencias 
que permitan afirmar que los estilos de aprendizaje influyen significativamente en el número de accesos de los estudiantes a los recursos digitales.

Con esos resultados es posible identificar que el uso de la teoría de los estilos de aprendizaje en el entorno virtual debe ser adaptado a una dinámica muy distinta del presencial. Los entornos virtuales facilitan el estudiante nuevas experiencias que se adaptan a las diversas características de los estilos. Para aún de eso es importante entender que los estilos de aprendizaje adaptados al virtual y direccionados para la forma como se utiliza esos espacios, son caracterizados como estilos del uso del espacio virtual. Barros (2013).

\section{REFERENCIAS BIBLIOGRÁFICAS}

Alonso, C., Gallego, D. \& Honey, P. (1999). Los estilos de aprendizaje: Procedimientos de diagnóstico y mejora (4th ed.). Bilbao: Ediciones Mensajero.

Alves, L., Barros, D. M. V., Okada, A. (2009). Moodle: Estratégias pedagógicas e estudo de caso. Salvador: Eduneb, p.394. Disponível online em: http://livromoodle.blogspot.com/

Barros, D. (2013) Estilos de aprendizagem e o uso das tecnologias. De facto editores: Santo Tirso, Portugal.

Bender, T. (2003). Discussion-based online teaching to enhance student learning: Theory, practice and assessment. Sterling, Virginia: Stylus Publishing, LLC.

Carol, C. (2015). Learning styles in Higher Education. A case study in History training. Procedia - Social and Behavioral Sciences 180, 256 - 261. https://doi.org/10.1016/.j.sbspro.2015.02.113

Cela, K., Fuertes, W., Alonso, C., \& Sánchez, F. (2010). Evaluación de herramientas web 2.0, estilos de aprendizaje y su aplicación en el ámbito educativo. Revista Estilos de Aprendizaje, 5(5), 117-134.

Creswell, J. (2014). Research design: Qualitative, quantitative, and mixed methods approaches (4th ed.). London: SAGE Publication Ltd.

Gallego, D. \& Alonso, C. (2010). Estilos de Aprendizaje. In J. Cué, J. Rineón, e M. Velázques Editors, IV Congreso Mundial de Estilos de Aprendizaje, pp. 24-48. México: México.

Gallego, D. (2013). Ya he diagnosticado el estilo de aprendizaje de mis alumnos y ahora qué hago? Revista de Estilos de Aprendizaje, 11(12), 1-15.

Graf, S., Liu, T., \& Kinshuk (2010). Analysis of learners' navigational behaviour and their learning styles in an online course. Journal of Computer Assisted Learning, 26, 116-131. https://doi.org/10.1111/i.13652729.2009.00336.x

Honey, P. \& Mumford, A. (1992). The manual of learning styles. Maidenhead: Peter Honey.

Hung, Y., Chang, R., Lin, C. (2016). Hybrid learning style identification and developing adaptive problem-solving learning activities. Computers in Human Behavior, 55, 552-561. https://doi.org/10.1016/i.chb.2015.07.004 
Estilos de aprendizaje de los estudiantes de la educación superior y el acceso a las herramientas de entornos virtuales

Küçük, M., Genç-Kumtepe, E. \& Tac, D. (2010). Support services and learning styles influencing interaction in asynchronous online discussions. Educational Media International, 47(1), March 2010, 39-56. https://doi.org/10.1080/09523981003654969

Kumar, R. (2011). Research methodology: A step-by-step guide for beginners. London: SAGE Publication Ltd.

Maroco, J. (2010). Análise estatística: Com utilização do SPSS (3. ${ }^{a}$ Ed.). Lisboa: Edições Sílabo.

Miranda, L., Morais, C., Goulão, F., \& Melaré, D. (2012). Estilos de aprendizagem: Uso do virtual por alunos do ensino superior. Revista de Estilos de Aprendizaje, 1(10), Octubre de 2012, 184-195.

Morais, C. (2000). Complexidade e comunicação mediada por computador na aprendizagem de conceitos matemáticos: Um estudo no 3.ำ ciclo do ensino básico. Tese de Doutoramento em Educação - Área do Conhecimento de Metodologia do Ensino da Matemática. Braga: Universidade do Minho.

Moreno, J. \& Defude, B. (2010). Learning styles and teaching strategies to improve the SCORM Learning Objects Quality. In Steimle, Jürgen (ed.) Proceedings of the 10th IEEE International Conference on Advanced Learning Technologies ICALT10, pp. 414-416, July 5-7, Sousse, Tunisia. https://doi.org/10.1109/icalt.2010.121

Özyurt, Ö. \& Özyurt, H.(2015). Learning style based individualized adaptive e-learning environments: Content analysis of the articles published from 2005 to 2014, Computers in Human Behavior, 52 (November 2015), 349358. https://doi.org/10.1016/i.chb.2015.06.020 Sílabo.

Pereira, A. (2004). Guia prático de utilização do SPSS. Lisboa: Edições

Rodríguez, A., Lozano, D., Aradillas, A., \& Duque, E. (2011). Uso de google docs como herramienta de construcción colaborativa tomando en cuenta los estilos de aprendizaje. Revista Estilos de Aprendizaje, 8 (8), 23-29.

Truong, H. (2016). Integrating learning styles and adaptive e-learning system: Current developments, problems and opportunities, Computers in Human Behavior, 55, 1185-1193. https://doi.org/10.1016/j.chb.2015.02.014

Zacharis, N. Z. (2011). The effect of learning style on preference for webbased courses and learning outcomes. British Journal of Educational Technology, 42(5), 790-800. https://doi.org/10.1111/j.1467-8535.2010.01104.x

Fatahi, S., Moradi, H., Kashani-Vahid, L. (2016). A survey of personality and learning styles models applied in virtual environments with emphasis on elearning environments, Artificial Intelligence Review, 46(3), 413-429. https://doi.org/10.1007/s10462-016-9469-7 
